\title{
A Study of Application Layer Paradigm for Lower Layer Energy Saving Potentials in Cloud-Edge Social User Wireless Image Sharing
}

\author{
Wei Wang \\ San Diego State University \\ 5500 Campanile Drive \\ San Diego, CA 92115 \\ 1-(619)-594-4302 \\ wwang@mail.sdsu.edu
}

\author{
Kazem Sohraby \\ South Dakota School of Mines and \\ Technology, 501 E St Joseph St \\ Rapid City, SD 57701 \\ 1-(605) 394-1219 \\ kazem.sohraby@sdsmt.edu
}

\begin{abstract}
Energy saving becomes critical in modern cloud wireless multimedia and mobile communication systems. In this paper we propose to study a new paradigm named application layer Position-Value diversity for wireless image sharing for cloudedge communications, which has significant energy saving potentials for modern wireless networking systems. In this new paradigm, saving energy is achieved by looking into application layer imaging traffic, in stead of MAC-PHY protocols at lower layers, and partitioning it into important positions and unimportant values. This paradigm could be integrated to existing wavelet-based tree compression, and truncation of image bit streams could be performed with regards to wireless communication energy budget estimation. Simulation results demonstrated that there are significant potentials of communication energy efficiency gain and Quality of Experience (QoE) enhancement in wireless image communication systems.
\end{abstract}

\section{Categories and Subject Descriptors}

C.2.1 [Network Architecture and Design]: Cross Layer Design

\section{General Terms}

Design.

\section{Keywords}

Wireless Multimedia Transmission, Energy Efficiency.

\section{INTRODUCTION}

The popularity of smart phone based mobile computing devices and ubiquitous availability of last-mile wireless cloud service access provide new opportunities of multimedia content sharing among smart phone users [1]. For example, users may frequently upload and download cloud image content through social media. However, the energy consumption for traffic intensive multimedia content sharing becomes a critical challenge.

In this paper we propose a new concept called application layer Position-Value diversity, which could potentially achieve significant energy efficiency gain in wireless multimedia communications in cloud edges. We specifically focus on transform domain (e.g. using wavelet and or cosine transforms to preprocess the images) position and value diversity instead of spatial domain (e.g. using quad tree structures to approximate the images) due to their effectiveness of concentrating picture information.

During the image compression process, the symbols and bits denoting the multimedia stream structures such as the wavelet zero tree root [2] [3] and tree structure nodes [4] [5] are of special interest in image compression, since their decoding determines the decoding of other parts of data such as tree leaves. Though video compression in not the focus of this paper, the motion compensation vectors and the reference macro blocks or code blocks are similar key information to be identified and marked as crucial position information. On the other hand, the symbols and bits denoting lighting values such as magnitude refinement in wavelet zero tree coding, and the residue quantization bits in video compression, could be organized into unimportant sections. The position-value separator will dispatch crucial position information and unimportant value information in separate queues, facilitating the allocation of lower layer unequal communication resources.

Research works have been reported in literature regarding prioritizing image or video packets to achieve efficiency transmission. The research in [6] explored the differences among multiple layers and proposed the application of different resource allocation strategies to different quality layers to improve distortion-latency performance. The research of [7] proposed a delay constrained wireless multimedia communication study in an Unequal Error Protection (UEP) fashion. The solution was derived by jointly adapting application layer multimedia packing and Medium Access Control (MAC) layer retransmission adaptation. Research [8] proposed a link layer network retry 
adaptation approach to enhance multimedia quality within the required latency deadline. Multiple quality layers in the media stream were treated with various Automatic Repeat reQuest (ARQ) retry limits, enhancing the efficiency of bandwidth resource allocation among the layers. More ARQ retries were assigned to the base layers to improve service quality, while fewer ARQ retries were assigned to quality enhancement layers to reduce bandwidth consumption. Additional research on delayconstrained quality optimization can be found in [9] [10].

However, most of above research are focusing on delay sensitive multimedia transmission to provide real-time services over wireless networks; energy efficiency considerations were largely ignored. In addition, multimedia packet prioritization was largely focused on providing various quality layers, but the finely detailed difference between position information and value information has been ignored. In our preliminary work [11] we have demonstrated energy saving potentials of assigning power and ARQ to different positions and values packets at PHY and MAC lower layers. In this paper we formalize the position and value diversity concept and explain the details of how to separate position and value information from application layer compression algorithms.

\section{Problem Statement}

A universal attribute of multimedia streams is unequal importance, as well as the inter dependency between compressed multimedia packets. Let $q$ denote the quality dependency of a coding unit (i.e. code word, or group of code words), $M$ denote the total number of coding units, and Si denote the set of coding units depending on qi. This position-value partitioning problem is equivalent to a selection/sorting problem, where each round of selection finds the "coding unit" with the highest $q$.

$$
q^{*}=\underset{i=1 \ldots M}{\arg \max }\left\{q_{i}+\sum_{j \in S_{i}} q_{j}\right\}
$$

From Equation 1 we can see, the packet importance is determined by both the individual contribution of each packet and the summative quality contribution of all dependents.

\section{Wavelet Tree Based Packet Prioritization}

In this section we proposed a new approach at application layer for multimedia position-value separation. The essential part of this proposed methodology is to separate crucial position information from the less important (unimportant) value information. This methodology could be designed as an add-in to the existing image compression process, exploring picture position and lighting value information diversity in the transform domain. In this paper we utilize a wavelet zero tree as a reference example to illustrate the partitioning process. As Figure 1 illustrates, the multimedia stream will first be processed by transforms, e.g. Discrete Cosine Transform (DCT) or Discrete Wavelet Transform (DWT). Next, the information will be concentrated in the upper-left corner of the image (or each picture in the video sequence) matrix. The quantization process will then be applied to the transform domain image matrix. In the compression process, the position and value information will be separated into different queues, entropy coded, and finally transmitted into the wireless channel.

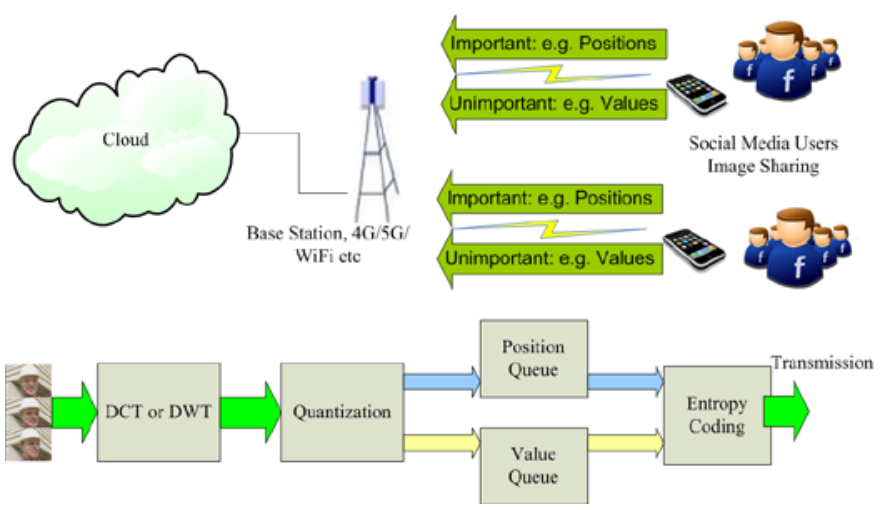

Fig 1. Position and value imaging sharing methodology at cloud edge

Every natural digital image is composed of the contours, edges, and lightening magnitude of each object. The human eye is generally sensitive to errors in contours and edges rather than errors in lightening magnitude. When DCT or DWT are applied to natural digital images, information is concentrated in the upperleft corner of the transform domain matrix. Next, bit-plane coding is applied to the transform domain coefficient matrix to produce a large number of "zeros" and a few magnitudes of those "large values." To reconstruct the original image with the closest approximation, the positions of these large values are much more important than their actual magnitudes. The question that remains is: what determines the positions of the large values? We expect the answer is: a large number of zeros. Run-length coding is typically applied in image compression, which efficiently presents large numbers of consecutive zero-runs with a simple numerical value. Alternatively, tree structures are also very effective in presenting the position information.

Thus, the position-value separation problem can be solved by using a "zero tree" to describe the position queue and "magnitudes leaves" to describe the value queue. We utilize a light weighted embedded zero tree wavelet scheme [12] as a reference example, and extend it into communication field, to see how the positions and values are fitted in the algorithm, as described in Algorithm 1.

Algorithm 1. Position and value partitioning based on embedded zero tree wavelet:

Step 0. Define I/O. Input: digital image I; Outputs: position queue $Q p$ and value queue $Q v$.

Step 1. Initialize source data and perform wavelet transform:

$$
X \Leftarrow W\{I, r, o\}
$$

where $X$ denotes the wavelet coefficient matrix, the $r$ denotes the wavelet transform levels, and $o$ denotes the default option for performing the wavelet transform using mode "db1".

Step 2. Find the bit-plane coding threshold $T$, which meets the following conditions:

$$
\begin{aligned}
& T=2^{i} \\
& \text { s.t. } \\
& 2^{i} \leq \max (I) \quad \text { and } \quad 2^{i+1} \geq \max (I)
\end{aligned}
$$


Run loops of Steps $3-4$ to quantize the wavelet coefficient matrix until $T$ meet termination condition.

Step 3. Scan the wavelet matrix with $k=1$ to $\operatorname{size}(I)$ do:

if $X(k) \geq T$

then $Q_{p}=Q_{p} \oplus[$ pos_k $]$; where $\oplus$ denote set

expansion;

record the large magnitude value $Q_{t}=Q_{t} \oplus[|X(k)|]$;

set currently location magnitude $X(k)$ to 0 ;

mark location pos_k as positive " 01 ” or negative “10" accordingly;

else

if $\mathrm{k}$ is in the DC wavelet coefficient frequency: mark location $\mathrm{k}$ as an isolated zero; else recursively check descendent $X(j)$ of ancestor $X(k)$ do

if $X(j) \geq T$ mark location pos_k as an isolated zero “ 11 ”; else

mark location pos_k as an zero tree root " 00 ”. end if; end if;

end if;

$T=T / 2$.

Step 4. Update the next bit of the bit-plane coding precision: for $\mathrm{k}=0$ to $\operatorname{size}(Q t)$ do

if $Q_{v}(i) \geq T$ then

put bit 1 to the end of the magnitude;

$Q_{v}(i)=Q_{v}(i) \oplus 1$

else

put bit 0 to the end of the magnitude;

$Q_{v}(i)=Q_{v}(i) \oplus 0$

end if;

end for.

Step 5. Output $Q p$ and value queue $Q v$.

\section{Prioritization Analysis for Energy Saving Potentials}

The communication and wireless transmission protocol that follows the multimedia source coding can be easily applied to the position and value queues, which have been prioritized with unequal treatment. Thus, energy and communication resources will be used more efficiently, and image quality will be improved without incurring excessive communication overhead. Intuitively, transmitting the packets with higher priority (i.e. is more energy efficient with regards to the following priority definition:

$$
p^{*}=\underset{i=1 \ldots N}{\arg \max }\left\{\frac{q_{i}}{e_{i}}\right\}
$$

where $p^{*}$ denotes the estimated priority, and $e$ denote the energy consumption of transmitting this packet.
In order for the packet to be successfully transmitted, certain protocols would be followed, such as performing a randomized back-off and waiting for acknowledgement confirmation. Let $P_{t x}$ denote the transmission power consumption, $P_{\text {elec }}$ denote the circuit power consumption, $P_{r x}$ denote the receive power consumption, $L$ denote the packet length, $R$ denote the transmission bit rate, $T_{\text {backoff }}$ denote the average back-off time, $T_{\text {SIFS }}$ and $T_{\text {DIFS }}$ denote the protocol specific short inter-frame spaces. The estimated energy consumption for a packet transmission could be approximated to:

$$
e=P_{t x} \frac{L}{R}+P_{\text {elec }} \times\left(T_{\text {backoff }}+T_{\text {DIFS }}\right)+P_{r} \times\left(T_{\text {SIFS }}+\frac{L_{\text {ack }}}{R}\right)
$$

From above analysis we can see, by analyzing the estimated energy consumption and packet quality contribution, the packet transmission priority could be dynamically determined within one image.

\section{Results and Discussion}

In this section we perform numerical analysis and experimental studies to see how effective the proposed packet prioritization could work with regards to the new position value diversity. We use a small picture with $128 * 128$ pixels as a simple example. Four levels of wavelet transform are performed using $\mathrm{db} 1$ wavelet. The transmission power is $16 \mathrm{dBm}$ similar to iPhone5 WiFi chipsets. The receive power and electronics power are assumed to be the same and transmission power, and no power scaling is applied. Basic transmission data rate is $1 \mathrm{Mbps}$. Assume CSMA/CA uses slot time of 20us with uniform random distribution back-off, MAC header length is 34 bytes, and the acknowledgement length is assumed to be 14 bytes. DIFS is 50us and SIFS is 10us.

Figure 2 shows the image quality with regards to progressive packet organization when more bit-planes are included in the bit stream. It is clear that more packets incorporated in the compressed bit stream could increase the overall quality perceived at the user side, i.e. the QoE. This baseline perform validation matches the widely accepted quality layer concept: when more bit-planes or quality layers are included in the image streams, users at the other end of the wireless channel could perceive higher quality gain, due to more fine details included in the transmitted bit stream. The odd numbers in this figure show the position packets and even numbers show the value packets.

Figure 3 and Figure 4 illustrate the individual packet quality contribution and the cumulative packet quality contribution respectively. From Figure 3 we can see, the position packets are generally more important than value packets, with higher Peak Signal to Noise Ratio (PSNR). The PSNR is measured as the difference by incorporating this packet or not in the decoding process, and thus reflect the contribution of QoE of each individual packet. Figure 4 illustrates the cumulative packet QoE contribution, i.e., if the packet is lost, how much quality 
degradation would be perceived by the user. Clearly the position packets show more importance compared with value packets, since the value packets are more isolated: when a value packet is lost in wireless channel, position packets are not impacted in decoding. On the other hand, when a position packet is lost, the avalanche effect of error propagation occurs during decoding process: the value packets and succeeding position packets in higher quality layers are all non-decodable since the locations of those wavelet coefficients are difficult to recover.

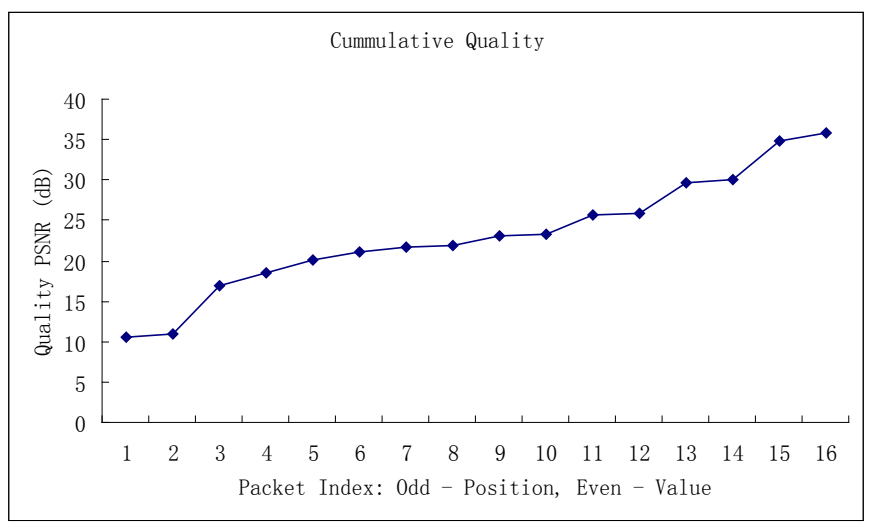

Fig 2. Quality gain with quality layer progression.

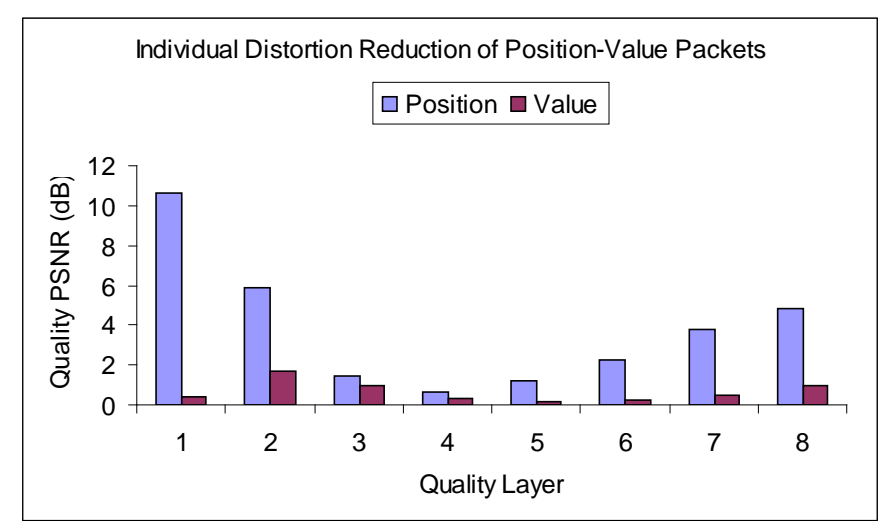

Fig 3. Individual packet quality contribution.

Figure 5 illustrates the wireless transmission cost in terms of packet lengths for each position and value packet. From this figure we can see two trends. First, packet sizes are typically higher when the quality layer increases. This is because the higher quality layers carry more fine details of the picture while the lower quality layers depict the rough picture information. Second, the lengths of position packets are generally shorter than the lengths of value packets. This is because the magnitude information is used to describe each coefficient and location information is used to depict those large coefficients.

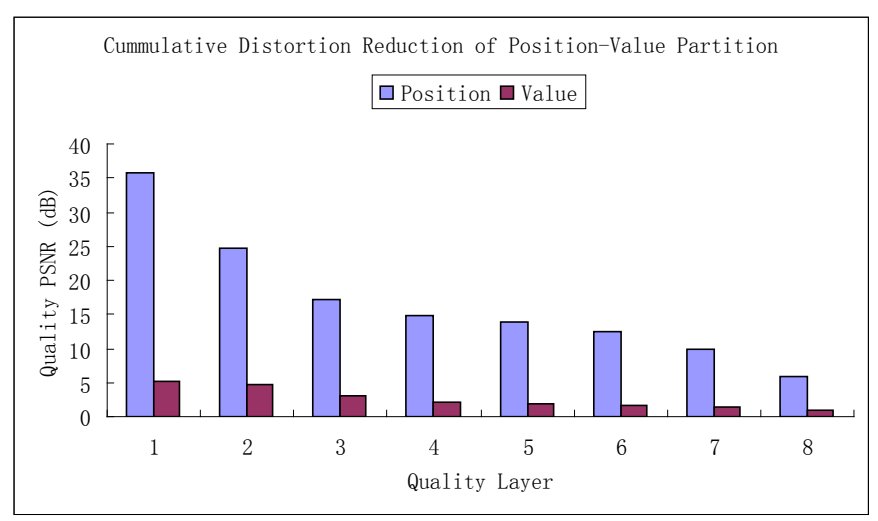

Fig 4. Cumulative packet quality contribution.

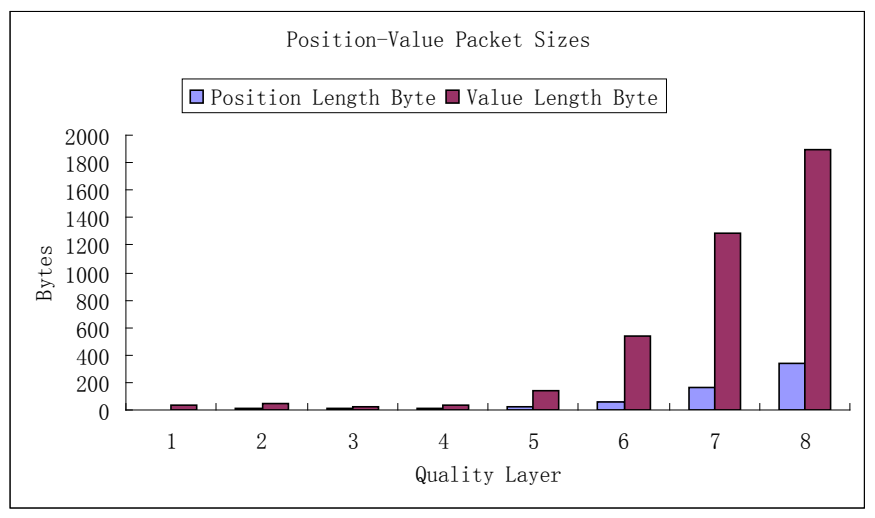

Fig 5. Individual packet size.

Figure 6 and Figure 7 show the normalized individual and normalized cumulative packet quality contribution, with consideration with packet length and wireless channel bandwidth. From both figures we can see, the position packets are much more important than those value packets. When wireless channel bandwidth or energy resources are limited, we should be able to prioritize position packets in lower quality layer for transmission.

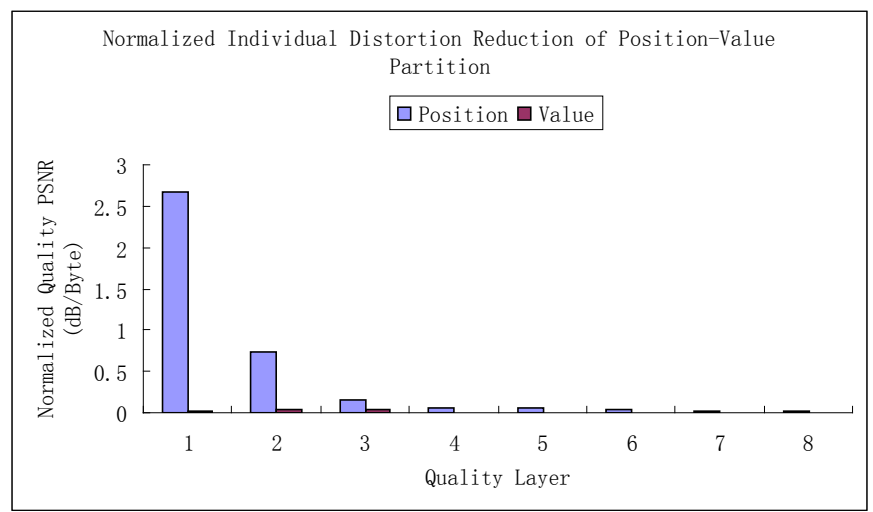

Fig 6. Normalize individual packet quality contribution. 


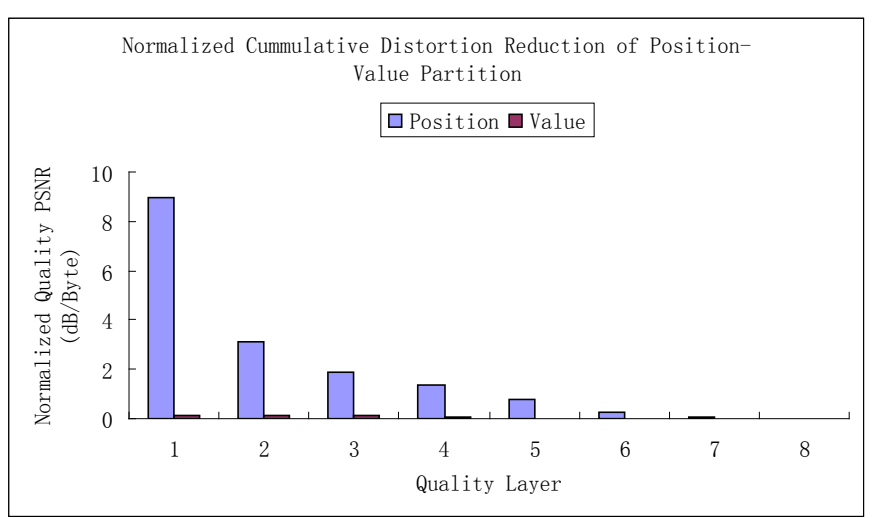

Fig 7. Normalize cumulative packet quality contribution.

Figure 8 shows the energy consumption of transmitting each position and value packet in the wireless channel in the $802.11 \mathrm{~b}$ environment. Typically the energy consumption is lower for transmitting position packets compared with transmitting value packets, since position packets are generally shorter. Due to the wireless protocol overheads, such difference decreases. Larger packets in high quality layers should more significant differences.

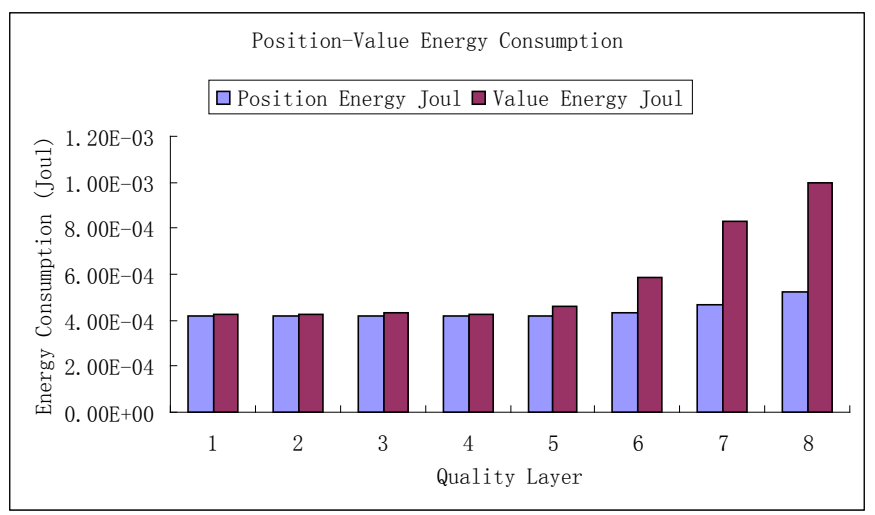

Fig 8. Individual packet energy consumption.

Figure 9 and Figure 10 show the individual and cumulative packet prioritization with regards to energy consumption. Either we consider each packet's individual QoE contribution, or the cumulative QoE contribution, position packets consistently show higher energy efficiency and QoE contribution. When we such diversity consider cumulatively, i.e. quantitatively how much the quality degradation would be when a position is lost in wireless channel, position packets show higher priority deserving more resources transmission. In other words, when we have limited energy or bandwidth resources, position packets in lower layer should have higher priorities in wireless image transmission.

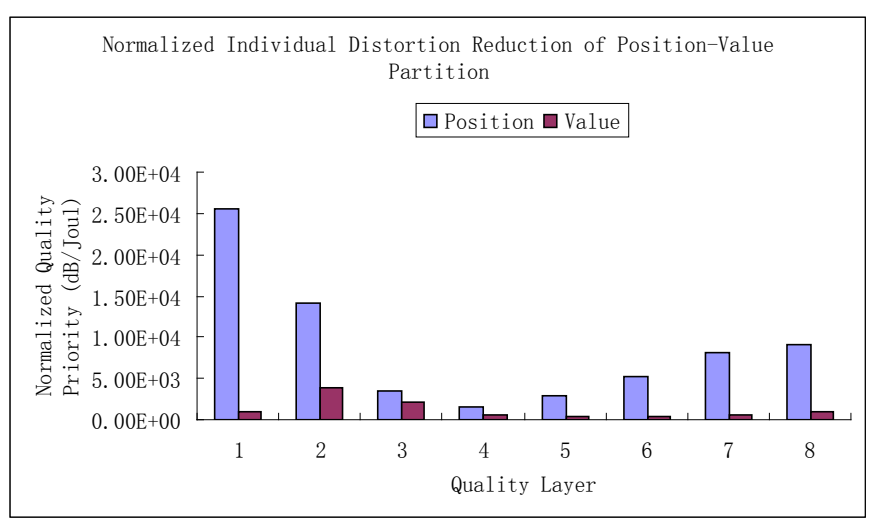

Fig 9. Normalize individual packet priority with energy consideration.

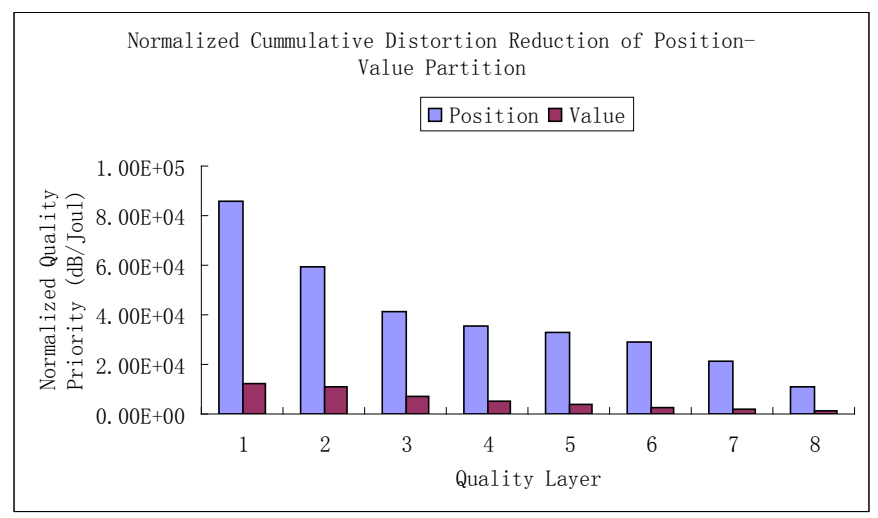

Fig 10. Normalize cumulative packet priority with energy consideration

\section{Conclusion}

In this paper we proposed to investigate the paradigm called position value diversity at application layer, which has considerable energy efficiency gain in wireless image sharing for cloud-edge mobile social user communication systems. Based on our preliminary study, we expect to be able to extract certain amount of position information from the image streams, which has significant impact on QoE for wireless image transmission. We also found the position packets are much more important than those value packets. When wireless channel bandwidth or energy resources are limited, we should be able to prioritize position packets in lower quality layer for transmission. Simulation results show that the new paradigm could achieve significant energy savings and quality improvements during wireless image content sharing process.

\section{ACKNOWLEDGMENTS}

This research in supported in part by National Science Foundation Grants No. 1463768 and No. 1423192 on energy efficient wireless multimedia communications.

\section{REFERENCES}

[1] B. Abu-Naim, W. Klas, "Smart authoring and sharing of multimedia content in personal area networks based on Subject of Interest," in 
Proc. IEEE Int. Conf. Multimedia and Expo Workshops, pp.1-6, Jul. 2014.

[2] M.W. Marcellin, A. Bilgin, "Quantifying the parent-child coding gain in zero-tree-based coders," IEEE Signal Processing Letters, vol. 8, no. 3, pp.67 - 69, 2001.

[3] Y. Cho, W.A. Pearlman, "Quantifying the Coding Performance of Zerotrees of Wavelet Coefficients: Degree-k Zerotree,” IEEE Trans. Signal Processing, vol. 55, no. 6, pp.2425 - 2431, 2007.

[4] O. Ghahabi, M.H. Savoji, "Modified EZW and SPIHT algorithms for perceptually audio and high quality speech coding," in Proc. IEEE Int. Conf. Signal and Image Processing Applications, pp.530 535, 2009.

[5] S. Dumitrescu, G. Rivers, S. Shirani, "Unequal Erasure Protection Technique for Scalable Multistreams," IEEE Trans. Image Processing, vol. 19, no. 2, pp.422 - 434, 2010

[6] M. van Der Schaar, N. Sai Shankar, "Cross-layer wireless multimedia transmission: challenges, principles, and new paradigms," IEEE Trans. Wireless Commun., vol.12, no. 4, pp.50 58, Aug. 2005

[7] P. Chou, Z. Miao, "Rate-distortion optimized streaming of packetized media," IEEE Trans. Multimedia., vol.8, no. 2, pp.390404, Apr.2006.
[8] L. Qiong, M. van der Schaar, "Providing adaptive QoS to layered video over wireless local area networks through real-time retry limit adaptation,” IEEE Trans. Multimedia, vol.6, no. 2, pp.278-290, Apr.2004.

[9] M. van der Schaar, D. Turaga, R. Wong, "Classification-based system for cross-layer optimized wireless video transmission," IEEE Trans. Multimedia, vol. 8, no. 5, pp.1082-1095, Oct.2006.

[10] M. van der Schaar, D.Turaga, "Cross-Layer packetization and retransmission strategies for delay-sensitive wireless multimedia transmission," IEEE Trans. Multimedia, vol.9, no.1, pp.185-197, Jan. 2007.

[11] W. Wang, D. Peng, H. Wang, H. Sharif, H. H. Chen, “EnergyConstrained Distortion Reduction Optimization for Wavelet-based Coded Image Transmission in Wireless Sensor Networks," IEEE Trans. Multimedia, vol. 10, no. 6, pp. 1169-1180, Oct. 2008.

[12] J. M. Shapiro, "Embedded Image Coding Using Zerotrees of Wavelet Coefficients," IEEE Trans. on Signal Processing, Vol. 41, No. 12, pp. 3445- 3462, Dec. 1993. 